\title{
Hamiltonicity of $k$-Traceable Graphs
}

\author{
${ }^{1}$ Frank Bullock, ${ }^{2}$ Peter Dankelmann, ${ }^{1}$ Marietjie Frick, \\ ${ }^{3}$ Michael A. Henning, ${ }^{4}$ Ortrud R. Oellermann; and ${ }^{1}$ Susan van Aardt ${ }^{\S}$ \\ ${ }^{1}$ University of South Africa, Pretoria \\ \{bullofes,Vaardsa\}@unisa.ac.za; marietjie.frick@gmail.com \\ ${ }^{2}$ University of KwaZulu-Natal, Westville Campus \\ dankelma@ukzn.ac.za \\ ${ }^{3}$ University of Johannesburg, Johannesburg \\ mahenning@uj.ac.za \\ ${ }^{4}$ University of Winnipeg, Winnipeg \\ o.oellermann@uwinnipeg.ca
}

Submitted: Jun 15, 2009; Accepted: Mar 2, 2011; Published: Mar 24, 2011

Mathematics Subject Classification: 05C38, 05C45

\begin{abstract}
Let $G$ be a graph. A Hamilton path in $G$ is a path containing every vertex of $G$. The graph $G$ is traceable if it contains a Hamilton path, while $G$ is $k$-traceable if every induced subgraph of $G$ of order $k$ is traceable. In this paper, we study hamiltonicity of $k$-traceable graphs. For $k \geq 2$ an integer, we define $H(k)$ to be the largest integer such that there exists a $k$-traceable graph of order $H(k)$ that is nonhamiltonian. For $k \leq 10$, we determine the exact value of $H(k)$. For $k \geq 11$, we show that $k+2 \leq H(k) \leq \frac{1}{2}(3 k-5)$.
\end{abstract}

Keywords: Hamiltonian graph; traceable; toughness

AMS subject classification: 05C38, $05 \mathrm{C} 45$

* Research supported in part by the South African National Research Foundation grant number 2053752

${ }^{\dagger}$ Research supported in part by the South African National Research Foundation

${ }^{\ddagger}$ Research supported in part by an NSERC grant Canada

${ }^{\S}$ Research supported in part by the South African National Research Foundation grant number TTK2004080300021 


\section{Introduction}

For notation and graph theory terminology we in general follow [14]. Specifically, let $G=(V, E)$ be a graph with vertex set $V$ of order $n=|V|$ and edge set $E$ of size $m=|E|$, and let $v$ be a vertex in $V$. The open neighborhood of $v$ is the set $N(v)=\{u \in V \mid u v \in E\}$. For a set $S$ of vertices, the open neighborhood of $S$ is defined by $N(S)=\cup_{v \in S} N(v)$. If $A$ and $B$ are subsets of $V(G)$, then we sometimes denote $N(A) \cap B$ by $N_{B}(A)$, and if $H$ and $J$ are subgraphs of $G$, then we write $N_{J}(H)$ for $N_{V(J)}(V(H))$. For a set $S \subseteq V$, the subgraph induced by $S$ is denoted by $G[S]$ while the graph $G-S$ is the graph obtained from $G$ by deleting the vertices in $S$ and all edges incident with $S$. If $S=\{v\}$, we simply denote $G-S$ by $G-v$ rather than $G-\{v\}$. We denote the degree of $v$ in $G$ by $d_{G}(v)$, or simply by $d(v)$ if the graph $G$ is clear from context. If $d_{G}(v)=n-1$, then $v$ is called a universal vertex of $G$. The minimum degree among the vertices of $G$ is denoted by $\delta(G)$. A cycle on $n$ vertices is denoted by $C_{n}$, while a path on $n$ vertices is denoted by $P_{n}$. We denote the number of components in a graph $G$ by $\operatorname{comp}(G)$.

Let $G$ be a graph. A Hamilton path in $G$ is a path containing every vertex of $G$. The graph $G$ is traceable if it contains a Hamilton path. If $G$ has a Hamilton path that starts at $x$ and ends at $y$, then $G$ is traceable from $x$ to $y$. If $G$ is traceable from each of its vertices, then $G$ is homogeneously traceable.

A Hamilton cycle in $G$ is a cycle containing every vertex of $G$. The graph $G$ is hamiltonian if it contains a Hamilton cycle. The graph $G$ is maximal nonhamiltonian, abbreviated $\mathrm{MNH}$, if $G$ is nonhamiltonian, but $G+e$ is hamiltonian for every edge $e \in E(\bar{G})$, where $\bar{G}$ denotes the complement of $G$. The graph $G$ is hypohamiltonian if $G$ is nonhamiltonian but $G-v$ is hamiltonian for every vertex $v$ in $G$.

A noncomplete graph $G$ is $t$-tough if $t \leq|S| / \operatorname{comp}(G-S)$ for every vertex cut $S \subset$ $V(G)$, where $t$ is a nonnegative real number. The maximum real number $t$ for which $G$ is $t$-tough is called the toughness of $G$ and is denoted by $t(G)$. Hence, if $G$ is not complete, then $t(G)=\min \{|S| / \operatorname{comp}(G-S)$, where the minimum is taken over all vertex cuts in $G$. By convention, the complete graphs have infinite toughness. An excellent survey of toughness in graphs has been written by Bauer, Broersma, and Schmeichel [2].

A graph is $k$-traceable if each of its induced subgraphs of order $k$ is traceable. Obviously, every graph is 1-traceable, while a graph is 2-traceable if and only if it is complete. Thus every 2 -traceable graph of order greater than 2 is hamiltonian. We extend this result to: every $k$-traceable graph of order greater than $k$ is hamiltonian, for each $k \in\{2,3,4,5,6,7\}$. This cannot be extended further, since the Petersen graph is a nonhamiltonian 8-traceable graph of order 10 .

We define $H(k)$ to be the largest integer such that there exists a nonhamiltonian $k$ traceable graph of order $H(k)$. It is easily seen that the minimum degree of a $k$-traceable graph of order $n$ is least $n-k+1$ and hence it follows from Dirac's well-known degree condition for hamiltonicity that for $k \geq 3$ every $k$-traceable graph of order at least $2 k-2$ is hamiltonian. On the other hand, for each $k \geq 1$ the path $P_{k}$ is a nonhamiltonian $k$ traceable graph of order $k$. These observations show that $H(k)$ is defined for every $k \geq 2$, 
and $k \leq H(k) \leq 2 k-3$. We determine the exact value of $H(k)$ for all $k \leq 10$, while for $k \geq 11$ we increase the lower bound for $H(k)$ to $k+2$ by constructing suitable graphs and we decrease the upper bound to $(3 k-5) / 2$ by combining known results on hamiltonicity with new results on $k$-traceable graphs.

\section{Known Results}

In this section, we list some known hamiltonicity results that we shall need in subsequent sections. We begin with the well-known theorem of Dirac [6].

Theorem 2.1 Let $G$ be a graph of order $n \geq 3$. If $\delta(G) \geq n / 2$, then $G$ is hamiltonian.

Jung [10] gave the following improvement of Dirac's Theorem for graphs that are 1tough.

Lemma 2.2 Let $G$ be a 1-tough graph of order $n \geq 11$. If $\delta(G) \geq \frac{1}{2}(n-4)$, then $G$ is hamiltonian.

The following result is a simple exercise in most graph theory textbooks.

Observation 2.3 Let $G$ be a graph and let $S$ be a nonempty proper subset of $V(G)$.

(a) If $G$ is hamiltonian, then $\operatorname{comp}(G-S) \leq|S|$.

(b) If $G$ is traceable, then $\operatorname{comp}(G-S) \leq|S|+1$.

Results due to Thomassen [13] and Doyen and van Diest [7] show that for all $n \geq 18$, there exists a hypohamiltonian graph with $n$ vertices. Aldred, McKay and Wormald [1] presented an exhaustive list of hypohamiltonian graphs on fewer than 18 vertices. Their list contains seven graphs, one each of orders 10, 13 and 15, and four of order 16. Hence we have the following existence result for hypohamiltonian graphs.

Theorem 2.4 There are no hypohamiltonian graphs of order $n$ for $n<10$ and for $n \in$ $\{11,12,14,17\}$. For all other values of $n$, there exists a hypohamiltonian graph of order $n$.

Chartrand, Gould and Kapoor [4] proved the following result.

Theorem 2.5 There exists a nonhamiltonian homogeneously traceable graph of order $n$ if and only if $n=2$ or $n \geq 9$.

In 1972, Chvátal and Erdős [5] proved the following relationship between the independence number and the connectivity of a nonhamiltonian graph.

Theorem 2.6 If $G$ is a nonhamiltonian graph, then $\alpha(G) \geq \kappa(G)+1$.

In 1979, Bigalke and Jung [3] showed that the following stronger result holds for 1-tough graphs with connectivity at least 3 .

Theorem 2.7 If $G$ is a 1-tough nonhamiltonian graph with $\kappa(G) \geq 3$, then either $G$ is the Petersen graph, or $\alpha(G) \geq \kappa(G)+2$. 


\section{Properties of $k$-traceable Graphs}

The following results show the relationships between the minimum degree, $\delta(G)$, the independence number, $\alpha(G)$, the connectivity, $\kappa(G)$, the toughness, $t(G)$, and the order, $n(G)$, of a $k$-traceable graph $G$.

Theorem 3.1 Let $G$ be a k-traceable graph of order $n$. Then, $G$ has the following properties.

(a) $\kappa(G) \geq n-k+1$.

(b) $\delta(G) \geq n-k+1$.

(c) If $k \geq 3$ and $n \geq 2 k-2$, then $G$ is hamiltonian.

(d) $\alpha(G) \leq\left\lceil\frac{k}{2}\right\rceil$ (and hence $k \geq 2 \alpha(G)-1$ ).

(e) If $n>k>2$, then $t(G) \geq \frac{2 n}{k+1}-1$.

(f) If $n>k>2$, then $G$ is 1-tough.

Proof. (a) Suppose $\kappa(G) \leq n-k$. Let $S$ be a vertex cut of $G$ with at most $n-k$ vertices. Then the graph $G-S$ is disconnected and has order at least $k$. Hence, $G$ has a disconnected induced subgraph of order $k$ and is therefore not $k$-traceable, a contradiction.

(b) This is immediate from part (a) and the fact that $\delta(G) \geq \kappa(G)$.

(c) Suppose $k \geq 3$ and $n \geq 2 k-2$ (and so, $n \geq 4$ ). Then, $n-k+1 \geq n / 2$, and so, by part (b), $\delta(G) \geq n / 2$. Hence, by Theorem 2.1, $G$ is hamiltonian.

(d) Suppose $\alpha(G) \geq\left\lceil\frac{k}{2}\right\rceil+1$. Let $X$ be an independent set of $\left\lceil\frac{k}{2}\right\rceil+1$ vertices of $G$. Now let $H$ be an induced subgraph of $G$ of order $k$ such that $X \subseteq V(H)$. Let $S=V(H) \backslash X$. Then, $\operatorname{comp}(H-S)=|X|=\left\lceil\frac{k}{2}\right\rceil+1 \geq\left\lfloor\frac{k}{2}\right\rfloor+1=|S|+2$, and so, by Observation 2.3, $H$ is nontraceable. Hence, $G$ is not $k$-traceable, a contradiction.

(e) We may assume $G$ is not a complete graph. Let $S$ be a vertex cut of $G$. Then $|S| \leq n-2$ and, by part (a), $|S| \geq n-k+1$. Let $r$ be defined by $|S|=n-k+r$, where $1 \leq r \leq k-2$.

Let $S^{\prime}$ be an $r$-element subset of $S$, and let $G^{\prime}=G-\left(S \backslash S^{\prime}\right)$. Then, $G^{\prime}$ is an induced subgraph of $G$ of order $k$. Since $G$ is $k$-traceable, the graph $G^{\prime}$ is traceable. Hence, by Observation 2.3(b), we have that $\operatorname{comp}(G-S)=\operatorname{comp}\left(G^{\prime}-S^{\prime}\right) \leq\left|S^{\prime}\right|+1=r+1$. But $|V(G)-S|=k-r$, so

$$
\operatorname{comp}(G-S) \leq \min \{r+1, k-r\}
$$

If $r \leq(k-1) / 2$, then $\min \{r+1, k-r\}=r+1$, so in this case

$$
\frac{|S|}{\operatorname{comp}(G-S)} \geq \frac{n-k+r}{r+1}=1-\frac{n-k-1}{r+1} \geq \frac{2 n}{k+1}-1 .
$$

If $r>(k-1) / 2$, then $\min \{r+1, k-r\}=k-r$, so in this case 


$$
\frac{|S|}{\operatorname{comp}(G-S)} \geq \frac{n-k+r}{k-r}=\frac{n}{k-r}-1>\frac{2 n}{k+1}-1
$$

Hence

$$
\min \left\{\frac{|S|}{\operatorname{comp}(G-S)}: S \text { a vertex cut of } G\right\} \geq \frac{2 n}{k+1}-1
$$

(f) This is an immediate consequence of part (e).

\section{Hamiltonicity of $k$-traceable graphs}

From Theorem 3.1(c) and the fact that the path $P_{k}$ is nonhamiltonian we obtain the following immediate lower and upper bounds for $H(k)$.

Observation $4.1 H(2)=2$, while $k \leq H(k) \leq 2 k-3$ for $k \geq 3$.

A hypohamiltonian graph of order $n$ is, clearly, $(n-1)$-traceable as well as $(n-2)$ traceable. Thus, $H(k) \geq k+2$ for every $k$ for which there exists a hypohamiltonian graph of order $k+2$. Thus as an immediate consequence of Theorem 2.4, we have that $H(k) \geq k+2$ for $k \in\{8,11,14\}$ and for $k \geq 16$. We show that, by "blowing up" a vertex of the Petersen graph, we can obtain, for each $k \geq 10$, a nonhamiltonian $k$-traceable graph of order $k+2$.

Lemma $4.2 H(k) \geq k+2$ for $k=8$ and for $k \geq 10$.

Proof. Let $P$ be the Petersen graph. Since $P$ is hypohamiltonian, it is 8 -traceable and 9-traceable. Hence $H(8) \geq 10$. Now let $k \geq 10$ and put $n=k+2$. Let $v \in V(P)$ and denote the neighbours of $v$ in $P$ by $v_{1}, v_{2}$ and $v_{3}$. Let $K$ be a complete graph of order $k-7$ and choose three distinct vertices, $w_{1}, w_{2}$, and $w_{3}$ in $K$. Let $P(n)$ be the graph of order $n$ obtained from the disjoint union of $P-v$ and $K$ by adding the three edges $v_{1} w_{1}$, $v_{2} w_{2}$ and $v_{3} w_{3}$. We show that $P(n)$ is a nonhamiltonian $k$-traceable graph.

Suppose that $P(n)$ has a Hamilton cycle $C$. Then, $C$ visits $K$ exactly once, since $K$ has only three vertices of attachment. We may therefore assume that $C$ intersects $K$ in a $w_{1}-w_{2}$ path $Q$. But then, replacing the subpath $v_{1} Q v_{2}$ in $C$ by the path $v_{1} v v_{2}$, produces a Hamilton cycle of $P$. This contradiction proves that $P(n)$ is nonhamiltonian.

We show next that $P(n)$ is $k$-traceable. It suffices to show that $P(n)-\{u, w\}$ is traceable for every two distinct vertices $u$ and $w$ of $P(n)$. Let $u$ and $w$ be an arbitrary pair of distinct vertices of $P(n)$.

Suppose that $u \notin V(K)$. Then, since $P$ is hypohamiltonian, $v$ lies on a Hamilton cycle, $C_{v}$, of $P-\{u\}$. Renaming vertices, if necessary, we may assume that $v_{1} v v_{2}$ is a subpath of $C_{v}$. Replacing this subpath in $C_{v}$ by the path $v_{1} Q v_{2}$, where $Q$ is a Hamilton path in 
$K$ that starts at $w_{1}$ and ends at $w_{2}$, produces a Hamilton cycle in $P(n)-\{u\}$. Removing the vertex $w$ from this cycle, produces a Hamilton path in $P(n)-\{u, w\}$. Similarly, if $w \notin V(K)$, then $P(n)-\{u, w\}$ is traceable.

Hence we may assume that $u \in V(K)$ and $w \in V(K)$. Renaming vertices, if necessary, we may assume that $w_{1} \notin\{u, w\}$. Since $P-v$ is hamiltonian, there is a Hamilton path $P_{v}$ in $P-v$ that ends at $v_{1}$. Let $P_{w}$ be a Hamilton path in $K-\{u, w\}$ that starts at $w_{1}$. Then, $P_{v} v_{1} w_{1} P_{w}$ is a Hamilton path in $P(n)-\{u, w\}$. Hence, $P(n)-\{u, w\}$ is traceable.

We remark that the nonhamiltonian $(n-2)$-traceable graph $P(n)$ of order $n$ constructed in the proof of Lemma 4.2 is only defined for $n \geq 12$.

Next we consider the existence of $k$-traceable graphs of order $k+1$. Skupien [12] calls a graph of order $n$ 1-traceable if it is $(n-1)$-traceable in our terminology. The following result is implied by Propositions 7.1 and 7.2 of [12]. We provide a proof for completeness.

Lemma 4.3 For a maximal nonhamiltonian graph $G$ of order $n \geq 3$ the following three statements are equivalent.

(1) G has no universal vertex.

(2) $G$ is homogeneously traceable.

(3) $G$ is $(n-1)$-traceable.

Proof. $(1) \Longrightarrow(2)$ : Suppose $G$ has no universal vertex. Let $u \in V(G)$. Then there is a vertex $v \in V(G)$ such that $u v \notin E(G)$. Since $G$ is MNH, this implies that $G+u v$ has a Hamilton cycle containing the edge $u v$. Hence, $G$ has a Hamilton path starting at $u$. Thus, $G$ is homogeneously traceable.

$(2) \Longrightarrow(3)$ : Suppose $G$ is homogeneously traceable. Let $H$ be an induced subgraph of $G$ of order $n-1$. Let $x$ be the vertex in $V(G) \backslash V(H)$. Then there is a Hamilton path $P$ of $G$ starting at $x$. But then $P-x$ is a Hamilton path of $H$, and so $H$ is traceable. Thus, $G$ is $(n-1)$-traceable.

$(3) \Longrightarrow(1)$ : Suppose $G$ is $(n-1)$-traceable. Let $x \in V(G)$. Then, $G-x$ has a Hamilton path $P$. Since $G$ is nonhamiltonian, $x$ is nonadjacent in $G$ to at least one of the two ends of $P$. Hence, $x$ is not a universal vertex of $G$. Thus, $G$ has no universal vertex.

As a consequence of Theorem 2.5 and Lemma 4.3, we have the following result.

Corollary $4.4 H(k) \neq k+1$ for $2 \leq k \leq 7$.

Proof. Suppose $G$ is a nonhamiltonian $k$-traceable graph of order $k+1$. Then $G$ is a subgraph of a MNH $k$-traceable graph of order $k+1$, so it follows from Theorem 2.5 and Lemma 4.3 that $k=1$ or $k \geq 8$.

The Chvátal-Erdős Theorem enables us to decrease the upper bound for $H(k)$ established in Observation 4.1.

Corollary $4.5 H(k) \leq \frac{3 k-3}{2}$ for $k \geq 3$. 
Proof. Let $G$ be a nonhamiltonian $k$-traceable graph of order $n \geq 3$. By Theorem 2.6, $\alpha(G) \geq \kappa(G)+1$. However, by parts (a) and (d) of Theorem 3.1, we have that $(k+1) / 2 \geq \alpha(G)$ and $\kappa(G) \geq n-k+1$. Hence, $(k+1) / 2 \geq n-k+2$, and so $n \leq(3 k-3) / 2$.

We now use the Bigalke-Jung Theorem, together with our results on the toughness, connectivity and independence number of $k$-traceable graphs, to further improve the upper bound when $k=7$ or $k \geq 9$.

Lemma 4.6 $H(k) \leq \frac{3 k-5}{2}$ for $k=7$ and for $k \geq 9$.

Proof. Suppose $G$ is a maximal nonhamiltonian $k$-traceable graph of order $n \geq k$, where $k=7$ or $k \geq 9$. If $n-k=1$, then, since $k \geq 7$, we have that $n=k+1 \leq(3 k-5) / 2$, and the desired result holds. Hence we may assume that $n-k \geq 2$. Thus, by Theorem 3.1(a), $\kappa(G) \geq n-k+1 \geq 3$. By Theorem 3.1(f), $G$ is 1-tough, and so by Theorem 2.7, either $G$ is the Petersen graph or $\alpha(G) \geq \kappa(G)+2$. But the Petersen graph has order 10 and is not 7 -traceable and we are assuming that $k \neq 8$. Hence, $G$ is not the Petersen graph. Thus, $\alpha(G) \geq \kappa(G)+2$. Thus, by Theorem 3.1(a), $\alpha(G) \geq n-k+3$. By Theorem 3.1, $(k+1) / 2 \geq \alpha(G)$. Hence, $(k+1) / 2 \geq n-k+3$, and so $n \leq(3 k-3) / 2$.

As a consequence of Observation 4.1, Lemma 4.2, Corollary 4.4, Corollary 4.5, and Lemma 4.6, we have the following summary of our results established thus far.

\section{Corollary 4.7 .}

(a) $H(k)=k$ if $2 \leq k \leq 7$.

(b) $H(8)=10$ and $10 \leq H(9) \leq 11$.

(c) $k+2 \leq H(k) \leq \frac{3 k-5}{2}$ if $k \geq 10$.

Proof. (a) It follows from Observation 4.1 and Corollary 4.5 that $H(k)=k$ for $k \in$ $\{2,3,4\}$ and that $5 \leq H(5) \leq 6$ and $6 \leq H(6) \leq 7$. Observation 4.1 and Lemma 4.6 imply that $7 \leq H(7) \leq 8$. But, by Corollary $4.4, H(k) \neq k+1$ for $k \in\{5,6,7\}$. Hence, $H(k)=k$ for $k \leq 7$.

(b) The Petersen graph shows that $H(8) \geq 10$ and $H(9) \geq 10$. Corollary 4.5 implies that $H(8) \leq 10$ and Lemma 4.6 implies that $H(9) \leq 11$.

(c) For $k \geq 10$ the lower bound follows from Lemma 4.2 and the upper bound from Lemma 4.6.

Corollary 4.7 shows that $H(9)$ is either 10 or $11, H(10)=12$ and $H(11)=13$ or 14 . Thus $H(k) \leq k+2$ for $k \leq 10$. We do not know whether there exists a $k$ such that $H(k)=k+1$ or such that $H(k)>k+2$. It therefore seems important to determine $H(9)$ and $H(11)$. The following lemma will prove useful, a proof of which is elementary and is omitted.

Lemma 4.8 If $S$ is an independent set of a path $P$, consisting of internal vertices of $P$, then $\left|N_{P}(S)\right| \geq|S|+1$. 
Corollary 4.9 Suppose $k$ is odd and $G$ is a $k$-traceable graph containing an independent set $I$ with $(k+1) / 2$ vertices. If $S \subseteq V(G) \backslash I$ such that $1 \leq|S| \leq(k-1) / 2$, then $\left|N_{I}(S)\right| \geq|S|+1$.

Proof. Let $H$ be any induced subgraph of $G$ such that $n(H)=k$ and $I \cup S \subseteq V(H)$. Then $H$ has a path $P$ of order $k$ that has both end-vertices in $I$ and alternates between $I$ and $V(H) \backslash I$. The result now follows from Lemma 4.8.

The following observation will prove useful.

Observation 4.10 Suppose a graph $G$ contains two disjoint paths $P:=v_{1} \ldots v_{k}$ and $Q:=x_{1} \ldots x_{r}$, with $k \geq 2$ and $r \geq 1$ such that $V(G)=V(P) \cup V(Q)$ and suppose $x_{1}$ and $x_{r}$ are adjacent to $v_{i}$ and $v_{j}$, respectively, where $1 \leq i<j \leq k$. Then $G$ is hamiltonian if it contains any of the following pairs of edges.

(a) $v_{1} v_{i+1}$ and $v_{k} v_{j-1}$.

(b) $v_{1} v_{j-1}$ and $v_{k} v_{i+1}$.

(c) $v_{1} v_{j-1}$ and $v_{k} v_{i-1}$.

(d) $v_{1} v_{j+1}$ and $v_{k} v_{i+1}$.

We are now in a position to determine the value of $H(9)$.

Theorem $4.11 H(9)=10$.

Proof. By Corollary 4.7, $10 \leq H(9) \leq 11$. We show that $H(9)=10$. Assume, to the contrary, that there exists a nonhamiltonian 9-traceable graph $G$ of order 11 (here $k=9$ and $n=11$ ). By Theorem 3.1(a), $\kappa(G) \geq 3$. By Theorem 3.1(f), $G$ is 1-tough, and so, by Theorem 2.7, $\alpha(G) \geq \kappa(G)+2 \geq 5$. By Theorem 3.1, $\alpha(G) \leq 5$. By Theorem 3.1(b), $\delta(G) \geq 3$. By Lemma 2.2, $\delta(G) \leq 3$. Hence, $\kappa(G)=\delta(G)=3$ and $\alpha(G)=5$.

Let $I$ be an independent set in $G$ with $|I|=5$. Then $V(G) \backslash I$ has six vertices and hence is not an independent set. Let $x_{1}, x_{2}$ be two adjacent vertices in $V(G) \backslash I$. Let $P: v_{1} v_{2} \ldots v_{9}$ be a Hamilton path of $V(G) \backslash\left\{x_{1}, x_{2}\right\}$. Then, $I=\left\{v_{1}, v_{3}, v_{5}, v_{7}, v_{9}\right\}$ and, by Corollary 4.9, $\left|N_{I}\left(x_{i}\right)\right| \geq 2$ for $i=1,2$ and $\left|N_{I}\left(\left\{x_{1}, x_{2}\right\}\right)\right| \geq 3$. We consider three cases, depending on $N\left(\left\{x_{1}, x_{2}\right\}\right) \cap\left\{v_{1}, v_{9}\right\}$.

Case 1. $N\left(\left\{x_{1}, x_{2}\right\}\right) \cap\left\{v_{1}, v_{9}\right\}=\emptyset$. Then $N_{I}\left(\left\{x_{1}, x_{2}\right\}\right)=\left\{v_{3}, v_{5}, v_{7}\right\}$. Since each of $x_{1}$ and $x_{2}$ has at least two neighbours in $I$, we may assume, without loss of generality, that $\left\{x_{1} v_{3}, x_{1} v_{5}, x_{2} v_{7}\right\} \subset E(G)$. We now consider two vertex-disjoint paths, namely the path $P$ defined earlier, and the path $Q: x_{1} x_{2}$. Since $\delta(G)=3, v_{9}$ is adjacent to at least one of $v_{4}$ and $v_{6}$. If $v_{4} v_{9} \in E(G)$, then, since $x_{1}$ and $x_{2}$ are adjacent to $v_{3}$ and $v_{7}$, respectively, Observation 4.10(b) and (d) imply that $v_{1}$ is nonadjacent to both $v_{6}$ and $v_{8}$. If $v_{6} v_{9} \in E(G)$, then, since $x_{1}$ and $x_{2}$ are adjacent to $v_{5}$ and $v_{7}$, respectively, Observation 4.10(b) and (d) once again imply that $v_{1}$ is nonadjacent to both $v_{6}$ and $v_{8}$. Hence, $N_{G}\left(v_{1}\right) \subseteq\left\{v_{2}, v_{8}\right\}$, and so $d_{G}\left(v_{1}\right) \leq 2$, contradicting the fact that $\delta(G)=3$. 
Case 2. $\left|N\left(\left\{x_{1}, x_{2}\right\}\right) \cap\left\{v_{1}, v_{9}\right\}\right|=1$.

We may assume that $N\left(\left\{x_{1}, x_{2}\right\}\right) \cap\left\{v_{1}, v_{9}\right\}=\left\{v_{1}\right\}$. Then $v_{2}$ has two neighbours $v_{i}$ and $v_{j}$ such that $i<j$ and $\{i, j\} \subset\{1,3,5,7\}$. By Observation 4.10, $v_{9}$ is nonadjacent to $v_{j-1}$. If $i \neq 1$, then $v_{9}$ is also nonadjacent to $v_{i-1}$, and so $d_{G}\left(v_{9}\right) \leq 2$, a contradiction. Hence, $i=1$. Since $\left|N_{I}\left(\left\{x_{1}, x_{2}\right\}\right)\right| \geq 3$, we may assume that $x_{1}$ is adjacent to $v_{t}$, where $t \neq j$ and $\{t, j\} \subset\{3,5,7\}$. Since $x_{1}$ and $x_{2}$ are adjacent to $v_{t}$ and $v_{1}$, respectively, Observation 4.10(a) implies that $v_{9}$ is nonadjacent to $v_{t-1}$. As observed earlier, $v_{9}$ is nonadjacent to $v_{j-1}$. Hence, $d_{G}\left(v_{9}\right) \leq 2$, a contradiction.

Case 3. $\left\{v_{1}, v_{9}\right\} \subseteq N\left(\left\{x_{1}, x_{2}\right\}\right)$.

Since $G$ is nonhamiltonian, we may assume that both $v_{1}$ and $v_{9}$ are adjacent to $x_{1}$ and nonadjacent to $x_{2}$. Then $v_{2}$ has two neighbours $v_{i}$ and $v_{j}$ such that $i<j$ and $\{i, j\} \subset$ $\{3,5,7\}$. Since $x_{1}$ and $x_{2}$ are adjacent to $v_{1}$ and $v_{i}$, respectively, Observation 4.10(a) implies that $v_{9}$ is nonadjacent to $v_{i-1}$. Further, since $x_{2}$ is adjacent to $v_{j}$, it follows that $v_{9}$ is nonadjacent to $v_{j-1}$. Since $x_{1}$ and $x_{2}$ are adjacent to $v_{9}$ and $v_{i}$, respectively, Observation $4.10(\mathrm{a})$ implies that $v_{1}$ is nonadjacent to $v_{i+1}$. Since $x_{2}$ is adjacent to $v_{j}$, it also follows that $v_{1}$ is nonadjacent to $v_{j+1}$. Let $r \in\{3,5,7\} \backslash\{i, j\}$. Since $\delta(G)=3$, $N_{G}\left(v_{9}\right)=\left\{v_{r-1}, v_{8}, x_{1}\right\}$ and $N_{G}\left(v_{1}\right)=\left\{v_{2}, v_{r+1}, x_{1}\right\}$.

Suppose that $\{i, j\}=\{3,5\}$. Then $r=7$ and $\left\{v_{6} v_{9}, v_{1} v_{8}\right\} \subset E(G)$. But then $v_{1} v_{8} v_{7} v_{6} v_{9}$ $x_{1} x_{2} v_{5} v_{4} v_{3} v_{2} v_{1}$ is a Hamilton cycle of $G$, a contradiction. Hence, $\{i, j\} \neq\{3,5\}$. By symmetry, $\{i, j\} \neq\{5,7\}$. Thus, $\{i, j\}=\{3,7\}$, and so $r=5$ and $\left\{v_{4} v_{9}, v_{1} v_{6}\right\} \subset E(G)$.

If $v_{5} x_{1} \in E(G)$, then $G$ is hamiltonian by Observation 4.10(c). If $v_{5} x_{2} \in E(G)$, then $G$ is hamiltonian by Observation $4.10(\mathrm{a})$. If $v_{5} v_{2} \in E(G)$, then $v_{5} v_{2} v_{3} x_{2} x_{1} v_{1} v_{6} v_{7} v_{8} v_{9} v_{4} v_{5}$ would be a Hamilton cycle of $G$. If $v_{5} v_{8} \in E(G)$, then $v_{8} v_{5} v_{4} v_{3} v_{2} v_{1} v_{6} v_{7} x_{2} x_{1} v_{9} v_{8}$ would be a Hamilton cycle of $G$. Since $G$ is nonhamiltonian, we therefore deduce that $v_{5}$ is adjacent only to $v_{4}$ and $v_{6}$. Hence, $d_{G}\left(v_{5}\right)=2$, a contradiction.

Since all three cases produce a contradiction, our assumption that $H(9)=11$ is incorrect. Hence, $H(9)=10$, as claimed.

As remarked earlier, Corollary 4.7 shows that $H(11)$ is either 13 or 14 . If there exists a nonhamiltonian 11-traceable graph $G$ of order 14, then, using our earlier results, $\kappa(G)=$ $\delta(G)=4$ and $\alpha(G)=6$. However we have yet to establish whether such a graph exists.

Our results are summarized in the following theorem.

Theorem 4.12 For $k \leq 10$, we have that

$$
H(k)= \begin{cases}k & \text { if } 2 \leq k \leq 7 \\ k+1 & \text { if } k=9 \\ k+2 & \text { if } k \in\{8,10\}\end{cases}
$$

while for $k \geq 11$,

$$
k+2 \leq H(k) \leq \frac{1}{2}(3 k-5)
$$




\section{The Circumference of $k$-Traceable Graphs}

If $C$ is a circumference cycle in a graph $G$ and $H$ is a component of $G-V(C)$, then obviously $\left|N_{C}(H)\right| \leq c(H) / 2$. We now show that this inequality is sharp if $G$ is $k$ traceable for some $k<n$.

Lemma 5.1 Suppose $G$ is a nonhamiltonian graph with circumference $c$ that is $k$-traceable for some $k<n$. If $C$ is a cycle in $G$ of length $c$ and $H$ is a component of $G-V(C)$, then $\left|N_{C}(H)\right|<c / 2$.

Proof. Suppose, to the contrary, that $H$ is a component of $G-V(C)$ such that $\left|N_{C}(H)\right| \geq$ $c / 2$. Then, since $N_{C}(H)$ does not contain two consecutive vertices of $C$, it follows that $\left|N_{C}(H)\right|=c / 2$ and $c$ is even. Let $C$ be the cycle $v_{1} v_{2} \ldots v_{c} v_{1}$.

First we show that $|V(H)|=1$. Suppose to the contrary that $|V(H)| \geq 2$. Then, since $\kappa(G) \geq 2$, there exist two vertices $v_{i}$ and $v_{i+2}$ on $C$ such that $v_{i} x, v_{i+2} y \in E(G)$ and $x \neq y$ with $x, y \in V(H)$. Let $P$ be an $x-y$ path in $H$. Replacing $v_{i} v_{i+1} v_{i+2}$ on $C$ with $v_{i} P v_{i+2}$ yields a cycle of order at least $c+1$. Hence $|V(H)|=1$ and we may assume that $V(H)=\{x\}$.

We show that there are at least two components in $G-V(C)$. Suppose to the contrary that $H$ is the only component of $G-V(C)$. Since $|V(H)|=1$, we have $c=n-1$. Then $\alpha \geq \frac{c}{2}+1$, since $V(G)-N_{C}(H)$ is an independent set. But now we obtain the contradiction $k \geq 2 \alpha-1 \geq c+1=n$. Hence there is at least one more component of $G-V(C)$, say $H^{\prime}$.

We now show that $N_{C}\left(H^{\prime}\right) \subseteq N_{C}(H)$. Suppose to the contrary, that there are adjacent vertices $v_{j}$ and $w$ with $v_{j} \in V(C), w \in V\left(H^{\prime}\right)$, and $v_{j} x \notin E(G)$. Since $\kappa(G) \geq 2$ there exists a vertex $u$ in $H^{\prime}$ which is adjacent to some vertex, $v_{i}$ say, of $C$, where $i \neq j$. Now let $P$ denote a $u-w$ path in $H^{\prime}$. Then $P$ is of order at least one and $|i-j| \geq 2$. Now if $v_{i} x \in E(G)$, then $v_{i} P v_{j} v_{j+1} \ldots v_{i-3} v_{i-2} x v_{j-1} v_{j-2} \ldots v_{i+1} v_{i}$ is a cycle of order at least $c+1$ and if $v_{i} x \notin E(G)$, then $v_{i} P v_{j} v_{j+1} \ldots v_{i-2} v_{i-1} x v_{j-1} v_{j-2} \ldots v_{i+1} v_{i}$ is a cycle of order at least $c+2$. Hence $N_{C}\left(H^{\prime}\right) \subseteq N_{C}(H)$.

Next we show that each component $H^{\prime} \neq H$ of $G-V(C)$ has only one vertex. Suppose to the contrary that $\left|V\left(H^{\prime}\right)\right| \geq 2$ and assume that $v_{i} w, v_{j} u \in E(G)$, where $v_{i}, v_{j} \in V(C)$ and $u, w \in H^{\prime}$ with $u \neq w$. Let $P$ denote a $u$-w path in $H^{\prime}$. Then $v_{i} v_{i+1} \ldots v_{j-3} v_{j-2} x v_{i-2} v_{i-3}$ $\ldots v_{j+1} v_{j} P v_{i}$ is a cycle of order at least $c+1$. Hence $H^{\prime}$ has only one vertex, and since $H^{\prime}$ was arbitrary we conclude that $V(G)-V(C)$ is an independent set.

But now $\alpha \geq \frac{c}{2}+n-c=n-\frac{c}{2}$. Hence $k \geq 2 \alpha-1 \geq 2 n-c-1$, and by $c \leq n-1$ we obtain the contradiction $k \geq n$.

We now establish an upper bound for the circumference of $k$-traceable graphs of order $n$ in terms of the difference between $n$ and $k$.

Theorem 5.2 Let $G$ be a connected, $k$-traceable graph of order $n>k \geq 2$. Then $c(G) \geq$ $\min \{n, 3(n-k)+3\}$. 
Proof. Suppose $G$ is not hamiltonian. Let $c$ be the circumference of $G$ and let $C=$ $v_{1} v_{2} \ldots v_{c} v_{1}$ be a longest cycle in $G$. Let $H_{1}, H_{2}, \ldots, H_{r}$ be the components of $G-V(C)$. For component $H_{1}$ let $A$ be the set of vertices of attachment in $C$, i.e., $A=N_{C}\left(H_{1}\right)$. Let $U$ be the set of successors of vertices of $A$ on $C$, and let $W$ be the set of predecessors of vertices of $A$ on $C$. We first note that $U$ and $W$ are distinct since otherwise $\left|N_{C}\left(H_{1}\right)\right|=c / 2$, contradicting Lemma 5.1. Let $R=V\left(H_{1}\right)$ and $S=\bigcup_{i>1} V\left(H_{i}\right)$.

The following standard argument shows that the set $U$ is independent, and that no two vertices of $U$ have neighbours in the same component of $G[S]$. Suppose this is false. Then there exist two vertices $v_{i}, v_{j} \in U$ and a $v_{i}-v_{j}$ path $P_{i, j}$ whose internal vertices are neither on $C$ nor in $H_{1}$. Vertices $v_{i-1}$ and $v_{j-1}$ are vertices of attachment of $H_{1}$, so they have neighbours $x$ and $y$, respectively, in $H_{1}$. Let $P_{x, y}$ be an $x$-y path in $H_{1}$. Now replacing the $v_{i-1}-v_{j}$ segment of $C$ with $v_{i-1} P_{x, y} v_{j-1} v_{j-2} v_{j-3} \ldots v_{i+1} P_{i, j}$ yields a longer cycle, contradicting the choice of $C$. The same statement holds for $W$. Clearly, there is no edge between $S$ and $R$ as their vertices are in different components of $G-V(C)$. Since no two consecutive vertices of $C$ are vertices of attachment of $H_{1}$, there is also no edge between $U \cup W$ and $R$. Hence we have the following:

(i) $U$ and $W$ are distinct independent sets.

(ii) $U \cup W, R, S$ are pairwise disjoint.

(iii) There is no edge joining $U \cup W \cup S$ to $R$.

(iv) No two vertices in $U$ (or $W$ ) have neighbours in the same component of $G[S]$. Consider the induced subgraph $F:=G[U \cup W \cup R \cup S]$. We claim that

$$
\operatorname{comp}(F) \geq|U \cap W|+2 \text {. }
$$

Let $F_{i}$ be a component of $F$. We first show that if $F_{i}$ contains a vertex in $U \cap W$, then it contains no other vertex in $U \cup W$. Indeed let $u_{1} \in U \cap W$ and suppose that $F_{i}$ contains a second vertex $u_{2} \in U \cup W, u_{2} \neq u_{1}$. Without loss of generality assume that $u_{2} \in U$. Then there exists a $u_{1}-u_{2}$ path in $F_{i}$. We may assume that there is no other vertex of $U \cup W$ on this path, and by (iii) the path contains no vertex of $R$. Since $u_{1}$ and $u_{2}$ are non-adjacent, it follows that each of $u_{1}$ and $u_{2}$ is adjacent to a vertex in $F_{i}$, contradicting property (iv). Hence $F_{i}$ contains no vertex in $U \cap W$ other than $u_{1}$.

Hence we have exactly $|U \cap W|$ components of $F$ that contain a vertex in $U \cap W$. Since $U \neq W$, the symmetric difference $U \Delta W$ is nonempty and there is at least one additional component of $F$ containing vertices of $U \Delta W$. Finally, by (iii), there is a further component containing vertices of $R$. In total we have at least $|U \cap W|+2$ components of $F$, which proves inequality (1).

Now choose a set $X$ of $|U \cap W|$ vertices in $V(G)-(U \cup W \cup R \cup S)$, for example from the $|U|$ vertices of attachment of $H_{1}$. Then

$$
|U \cup W \cup R \cup S \cup X|=|U \cup W|+|R|+|S|+|U \cap W|=|U|+|W|+|R|+|S| .
$$

But $U \cup W \cup R \cup S \cup X$ is not traceable, since removing the $|U \cap W|$ vertices in $X$ yields a graph with at least $|U \cap W|+2$ components. Moreover, $U \cup W \cup R \cup S \cup X$ contains a 
non-traceable subset of order $i$ for all $i \in\{2,3, \ldots,|U|+|W|+|R|+|S|\}$. Hence we have $k>|U|+|W|+|R|+|S|$, as desired.

Now $G$ is $(n-k+1)$-connected, so we have $|A|=|U|=|W| \geq n-k+1$. Also, $|R|+|S|=n-c(G)$ since $C$ is a longest cycle in $G$. Hence $k \geq 2(n-k+1)+n-c(G)+1$, or, equivalently, $c(G) \geq 3(n-k)+3$, as desired.

The Petersen graph is an example of a nonhamiltonian graph realizing the bound on the circumference given in Theorem 5.2 (since it is 8-traceable).

\section{Acknowledgment}

The authors wish to thank UNISA and the NRF of SA for their sponsorship of two workshops held at Salt Rock (11-15 March 2007 and 7-9 November 2008) where most of the joint research for this paper was conducted.

\section{References}

[1] R. E. L. Aldred, B. D. McKay and N. C. Wormald, Small hypo-Hamiltonian graphs. J. Combin. Math. Combin. Comput. 23 (1997), 143-152. (or see http://cs.anu.edu.au/ bdm/papers/hypo.pdf).

[2] D. Bauer, H. Broersma, and E. Schmeichel, Toughness in Graphs-A Survey. Graphs Combin. 22 (2006), 1-35.

[3] A. Bigalke and H. A. Jung, Über Hamiltonsche Kreise und Unabhängige Ecken in Graphen, Monatsh. Math. 88 (1979) 195-210.

[4] G. Chartrand, H. J. Gould and S. F. Kapoor, On homogeneously traceable nonhamiltonian graphs. 2nd Internat. Conf. on Combinatorics, Math. Ann. N.Y. Acad. Sci. 319 (1979), 130-135.

[5] V. Chvátal and P. Erdős, A note on hamiltonian circuits. Discrete Math. 2 (1972), 111-113.

[6] G. A. Dirac, Some theorems on abstract graphs. Proc. London Math. Soc. 2 (1952), 69-81.

[7] L. Doyen and V. van Diest, New families of hypohamiltonian graphs. Discrete Math. 13 (1975), 225-236.

[8] D. A. Holton and J. Sheehan, The Petersen Graph. Australian Mathematical Society Lecture Series, 7. Cambridge University Press, Cambridge, 1993.

[9] M. Grötschel, C. Thomassen and Y Wakabayashi, Hypotraceable digraphs, J. Graph Theory 4 (1980) 377-381.

[10] H. A. Jung, On maximal circuits in finite graphs. Ann. Discrete Math. 3 (1978), $129-144$.

[11] Z. Skupien, Homogeneously traceable and hamiltonian connected graphs. Demonstratio Mathematica 17 No.4 (1984), 1051-1067. 
[12] Z. Skupien, Some examples in hamiltonian graph theory. Bull. Polish Acad. Sciences Math. 36 No.5-6 (1988), 363-374.

[13] C. Thomassen, Hypohamiltonian and hypotraceable graphs. Discrete Math. 9 (1974) 91-96.

[14] D. B. West, Introduction to Graph Theory: Second edition, Prentice-Hall, Upper Saddle River, NJ (2001). 